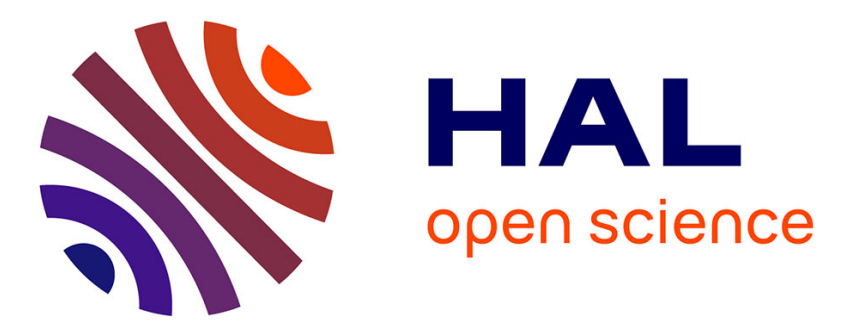

\title{
Nouveau sondage sur le site paléolithique des Petits Guinards à Creuzier-le-Vieux (Allier, France) : des données inattendues
}

Laure Fontana, Laurent Lang, François-Xavier Chauvière, Marcel Jeannet, Lionel Magoga

\section{To cite this version:}

Laure Fontana, Laurent Lang, François-Xavier Chauvière, Marcel Jeannet, Lionel Magoga. Nouveau sondage sur le site paléolithique des Petits Guinards à Creuzier-le-Vieux (Allier, France) : des données inattendues. Bulletin de la Société préhistorique française, 2003, 100 (3), pp.591-596. 10.3406/bspf.2003.12875 . hal-02539191

\section{HAL Id: hal-02539191 \\ https://hal.science/hal-02539191}

Submitted on 9 Apr 2020

HAL is a multi-disciplinary open access archive for the deposit and dissemination of scientific research documents, whether they are published or not. The documents may come from teaching and research institutions in France or abroad, or from public or private research centers.
L'archive ouverte pluridisciplinaire HAL, est destinée au dépôt et à la diffusion de documents scientifiques de niveau recherche, publiés ou non, émanant des établissements d'enseignement et de recherche français ou étrangers, des laboratoires publics ou privés. 


\author{
Laure Fontana \\ Laurent Lang \\ François-Xavier Chauvière \\ Marcel Jeannet \\ Lionel Magoga
}

\title{
Nouveau sondage sur le site paléolithique des Petits Guinards à Creuzier-le-Vieux (Allier, France) : des données inattendues
}

In: Bulletin de la Société préhistorique française. 2003, tome 100, N. 3. pp. 591-596.

Citer ce document / Cite this document :

Fontana Laure, Lang Laurent, Chauvière François-Xavier, Jeannet Marcel, Magoga Lionel. Nouveau sondage sur le site paléolithique des Petits Guinards à Creuzier-le-Vieux (Allier, France) : des données inattendues. In: Bulletin de la Société préhistorique française. 2003, tome 100, N. 3. pp. 591-596.

doi : 10.3406/bspf.2003.12875

http://www.persee.fr/web/revues/home/prescript/article/bspf_0249-7638_2003_num_100_3_12875 


\title{
ACTUALITÉS SCIENTIFIQUES
}

\author{
DÉCOUVERTES RÉCENTES
}

\section{Nouveau sondage sur le site paléolithique des Petits Guinards à Creuzier-le-Vieux (Allier, France) : des données inattendues.}

Résumé : Identifié comme un site de plein air, en bord d'Allier, les découvertes anciennes sur le site des Petits Guinards avaient attribué ses occupations au Magdalénien supérieur alors qu'une date radiocarbone indiquait plutôt une présence badegoulienne. Une campagne de sondages a permis de réévaluer l'importance de ce site et de mieux comprendre sa nature. L'étude stratigraphique et taphonomique du sondage principal permet d'identifier ces dépôts comme secondaires, liés à un glissement en masse de type loupe de glissement, et de situer l'emplacement originel des occupations à une centaine de mètres en contre-haut, au pied d'un escarpement calcaire. Les déterminations préliminaires des nombreux restes de macrofaune très bien conservés, mettent en évidence des chasses au Renne et au Cheval qui se sont déroulées durant le printemps. Les dizaines de milliers de restes de microfaune attestent en partie de la localisation du site en pied d'escarpement tandis que le cortège des espèces identifie globalement des conditions climatiques continentales très rigoureuses. Les restes d'industrie lithique, nettement moins nombreux, identifient cet assemblage à forte composante lamellaire comme typique d'un Magdalénien stricto sensu. Les objets sur matières dures animales constituent l'assemblage le plus inattendu : pointes de projectile, ciseaux décorés et autres objets, plutôt rares ou mêmes inédits pour le Massif central. Les quatre premières datations radiocarbones se situent autour de $13500 \mathrm{BP}$ pour les deux tiers des niveaux supérieurs alors que la couche la plus profonde est datée d'environ $18000 \mathrm{BP}$.

Abstract: Mentioned as an open-air site, along the Allier river, ancient studies had identified the occupations of the "Petits Guinards" site as Upper Magdalenian even though a radiocarbon date indicated instead the Badegoulian period. Following a field season where testpits were carried out, the nature of the site and its importance can now be reassessed and better understood. Stratigraphic and taphonomic studies of the main excavation lead us to consider these deposits as secondary, in relation with an earth slump and the original location of the site to be hundred meters up the slope, at the bottom of a limestone outcrop. Preliminary determinations of the numerous remains of macrofauna, very well preserved, document Reindeer and Horse hunting, which took place during the spring. Thousands of remains of microfauna bear witness to this location at the bottom of the limestone cliff. The identified species of microfanna are correlated to a very rigorous continental climate. The remains of lithic industry, which are less abundant, correspond to a typical Magdalenian assemblage, with a high component of bladelets. The bone tool industry is the most surprising: projectile points, engraved chisels and other artefacts, rather rare or even unknown in the Massif Central. The first four dates are around 13,500 uncal.BP for the two-thirds of upper levels whereas the lowest level is dated to 18,000 BP.

\section{Introduction}

Le site des Petits Guinards se trouve en bord d'Allier, à $3 \mathrm{~km}$ au nord de Vichy, à une altitude de $260 \mathrm{~m}$. Entre 1981 et 1985, l'un d'entre nous (L.M.) effectua des ramassages dans le talus et un sondage qui livrèrent une majorité de restes fauniques, de l'industrie lithique et une aiguille à chas (Magoga, 1985 ; Mazière, 1982, 1984. 1986). Cette accumulation de vestiges dans le talus fut interprétée comme un dépôt en position secondaire, dont l'origine était liée à une coulée de solifluxion. Une datation radiocarbone, réalisée sur un lot de $400 \mathrm{~g}$ d'ossements, livra une date de $17420 \pm$ $330 \mathrm{BP}$ (Ly 2737) alors que l'industrie lithique (burins dièdres et lamelles à dos) avait été attribuée à un Magdalénien supérieur. Nous avons entrepris, durant l'automne 2002, trois campagnes de sondages dont l'objectif était multiple : confirmer la position secondaire des dépôts; tenter d'identifier précisément l'endroit où des parties du site étaient conservées; 
réaliser des observations stratigraphiques afin de comprendre la dynamique des dépôts; mettre au jour des vestiges fauniques et lithiques afin de préciser les modalités des occupations humaines et leur attribution chrono-culturelle. Après avoir effectué plusieurs sondages infructueux à l'intérieur de la parcelle et recueilli plusieurs restes dans le talus qui borde la route, nous avons entrepris un sondage de $14 \mathrm{~m}^{2}$ dans le talus même, sur une épaisseur de $60 \mathrm{~cm}$, avant de choisir une bande de $3,50 \mathrm{~m}^{2}$ qui a été fouillée jusqu'à une profondeur d' $1,60 \mathrm{~m}$. Plusieurs milliers de restes proviennent de ce sondage. Il s'agit en grande majorité de restes fauniques (macromammifères, micromammifères, avifaune, ichtyofaune, herpétofaune), étroitement associés à des restes d'industrie lithique et d'objets sur matières dures animales.

\section{Mise en place des dépôts}

Le site se trouve sur une formation complexe de versants composés de matériaux argilo-calcaires et de sables, "affectés par une intense solifluxion" (carte géologique de Vichy, p. 30), comme un certain nombre de secteurs en rive droite de l'Allier au modelé très particulier.

Le premier objectif des sondages était d'identifier l'emplacement originel de l'installation et d'appréhender, de façon préliminaire les processus dynamiques et diagénétiques qui ont participé à la formation du site en évaluant plus particulièrement le degré des perturbations.

Deux coupes stratigraphiques ont été relevées et si plusieurs ensembles stratigraphiques d'épaisseur variable ont été identifiés, les dépôts présentent une certaine homogénéité. Il s'agit de limons bruns à granules de calcaire avec quelques blocs de taille variable et d'une longueur maximale de $40 \mathrm{~cm}$ (exception faite de quelques énormes blocs). La base des dépôts est située à $1,70 \mathrm{~m}$ de profondeur. Si le mobilier est réparti sur toute l'épaisseur de la stratigraphie, sa densité augmente et la conservation des restes fauniques devient excellente à partir de $60 \mathrm{~cm}$ de profondeur. De plus, cette densité nouvelle et cet état de surface s'accompagnent de la présence systématique des restes osseux et dentaires de micromammifères, en quantités très importantes. Enfin, des restes d'os brûlés, des pierres rubéfiées et des charbons diffus ont été trouvés en grande quantité.

Plusieurs données nous ont permis d'appréhender la question de la mise en place des dépôts et d'argumenter la position secondaire des témoins anthropiques (Fontana et al., 2003). Si toutes les caractéristiques mises en évidence confirment le caractère secondaire de ces dépôts, aucune en revanche n'identifie une quelconque solifluxion (ni une coulée de débris). Les observations réalisées sur le type de fracturation et sur l'état des surfaces osseuses des restes de macrofaune et de microfaune, ainsi que sur les blocs et l'état de surface des silex témoignent toutes dans le même sens : la solifluxion ne semblant pas être à l'origine de ce déplacement, l'hypothèse la plus plausible aujourd'hui est celle d'un déplacement en masse, rapide, de type loupe de glissement.

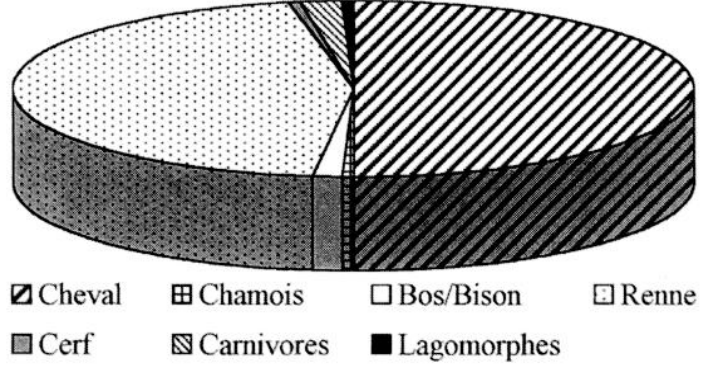

Fig. 1 - Taxons identifiés aux Petits Guinards (en \% du NR évalué). Identified taxa : Les Petits Guinards (\% of estimated NISP).

\section{Les restes fauniques}

L'étude en cours des restes fauniques documente des aspects paléoenvironnementaux et économiques qui sont de la première importance dans ce secteur très mal connu, quelle que soit la période.

Environ 5000 restes de macrofaune constituent ce premier assemblage au sein duquel 13 taxons au moins sont représentés (fig. 1) : des herbivores (le Renne, le Cerf, un grand boviné, le Chamois, le Cheval, un petit bovidé indéterminé, un Lièvre et le Lapin) et des carnivores (le Renard commun, le Loup, un ursidé, l'Hermine et la Belette).

Du point de vue de la caractérisation des chasses et de l'occupation, l'étude archéozoologique préliminaire a déjà pu répondre à certaines interrogations (Fontana et al., 2003). Ce site témoigne d'une chasse probablement concomitante au Renne et au Cheval et les saisons de chasse s'étalent entre les mois de mars et de juillet. Enfin, les premières caractéristiques mettent déjà en évidence des ressemblances importantes avec les données du Pont-de-Longues (Fontana, 2000).

La microfaune est abondante et son état de conservation est excellent. Ces milliers de restes ne peuvent provenir (au moins pour une partie d'entre eux) que de pelotes de réjection de rapaces. Une vingtaine d'espèces ont été identifiées à partir des 38000 restes estimés, appartenant majoritairement aux campagnols (neuf espèces), au Souslik (ou Spermophile) et à la Taupe. La bonne représentation du Campagnol nordique et du Campagnol des hauteurs, associé au Souslik et au Lemming à collier (et dans une moindre mesure au Grand Hamster, à la Siciste des bouleaux et à la Musaraigne carrelet) identifie des conditions climatiques continentales particulièrement rigoureuses comme celles du Dryas ancien. Cette accumulation de restes de micomammifères, caractérisée par une sur-représentation des individus sub-adultes semble être due, dans l'état actuel de l'étude, à l'action d'au moins deux types de prédateur, rapaces et carnivores, qui sont en cours d'identification.

Parmi la trentaine de restes d'ichtyofaune mise au jour, on note la présence de salmonidés et de cyprinidés dont les prédateurs restent à déterminer (étude en cours M. Sternberg). De même, l'identification des restes d'oiseaux (étude en cours C. Mourer-Chauviré) contribuera à l'étude taphonomique globale liée à l'origine des accumulations. 


\section{Les restes humains}

Une phalange humaine a été identifiée comme appartenant à un individu jeune. Elle constitue la seconde découverte de ce type puisqu'une molaire humaine d'adulte se trouvait parmi les restes fauniques de la collection ancienne.

\section{L'industrie lithique}

Elle est exclusivement constituée de silex, exception faite de cinq fragments de quartz laiteux qui ne présentent pas de stigmates évidents de taille intentionnelle. Les variétés de silex translucides à grain très fin, le plus souvent de couleur blonde mais aussi parfois grise à noire, sont de loin les mieux représentées (plus de $80 \%$ de l'industrie). Ces matériaux, bien représentés dans tous les assemblages du Paléolithique supérieur d'Auvergne, proviennent de formations du crétacé supérieur, plus précisément de dépôts crayeux du Turonien du Berry ou de Touraine (Masson, 1981; Surmely et al., 2002), distants de $200 \mathrm{~km}$ environ.

Sur les 692 éléments d'industrie lithique, près de la moitié sont des esquilles (éclats de moins d' $1 \mathrm{~cm}$ ), recueillies principalement lors du tamisage des déblais. La composante lamellaire de l'assemblage (esquilles exclues) est très élevée. Un seul nucléus (à lamelles) est présent, voire deux si on considère un burin transversal à enlèvements lamellaires multiples (fig. 2, $\left.n^{\circ} 11\right)$ comme un nucléus. De manière générale, les éléments de l'industrie sont de dimensions réduites, aucun n'atteignant six $\mathrm{cm}$ dans sa plus grande dimension. Le sondage a livré 67 outils, ce qui correspond à près de $10 \%$ du mobilier lithique recueilli (esquilles comprises). Si on exclut les esquilles du décompte, l'outillage représente environ $18 \%$ de l'industrie. Ce taux de transformation élevé des produits de débitage en outils est à mettre probablement en relation avec l'éloignement des sources d'approvisionnement en matières premières. Plus des deux tiers des outils sont façonnés sur des supports en silex blond translucide (71\%). Au sein des microlithes les lamelles à dos constituent la classe d'outils de loin la mieux représentée, avec 41 exemplaires (fig. $2, \mathrm{n}^{\circ} 3$ à 6). Elles se caractérisent par une importante variabilité dimensionnelle, due à la présence de nombreux éléments de très petites dimensions. Deux triangles scalènes sont présents (fig. $2, \mathrm{n}^{\circ} \mathrm{l}$ et 2 ). Parmi les outils du fonds commun, les sept burins constituent la seconde classe d'outils, à égalité avec les lames tronquées et les chutes de burins sont bien représentées avec 41 individus. Les burins dièdres sont au nombre de quatre. Les lames tronquées correspondent généralement à des fragments de lames de modules variés portant des troncatures directes plus ou moins obliques. Un seul perçoir est présent. La série comporte deux pièces esquillées, dont une sur éclat présente une retouche abrupte d'un bord. Les cinq lames retouchées sont toutes fragmentées : certaines peuvent correspondre à des fragments d'outils appartenant à d'autres types (burins, grattoirs...). L'absence de grattoirs est probablement imputable à la faiblesse numérique de l'outillage hors microlithes, la récolte de 1981-1982 en ayant livré. Bien que l'assemblage recueilli soit limité en raison de la surface réduite du sondage, il a été possible d'appréhender les principales caractéristiques technologiques de l'industrie (Fontana et al., 2003).

\section{L'industrie sur matières dures animales}

Plusieurs dizaines de fragments de bois de Renne (bois de massacre et de chute) ont été récoltés. Certains, qui constituent autant de déchets de fabrication, portent des traces évidentes d'un débitage par percussion lancée ou d'un léger raclage. Les objets finis sont au nombre de six. Ils peuvent être répartis en deux catégories fonctionnelles distinctes : des pièces intermédiaires de type ciseau, et des pointes de projectiles. Les pièces intermédiaires de type ciseau sont au nombre de trois dont l'un porte un décor élaboré, constitué de chevrons et d'incisions parallèles profondes (fig. $3, n^{\circ} 1$ ). Trois autres pièces, toutes fragmentaires, sont interprétées comme des pointes de projectile et ont été façonnées par raclage sur la totalité de leur volume (fig. $3, \mathrm{n}^{\circ} 2$ ). Un seul élément travaillé sur os a été identifié. Il s'agit d'une extrémité proximale de métacarpien d'un petit bovidé qui porte les stigmates d'un rainurage multiple destiné à la production de baguettes rectilignes, supports utilisés dans la fabrication des aiguilles à chas (fig. $3, \mathrm{n}^{\circ} 3$ ). Enfin, la racine d'une incisive de cervidé ou de petit bovidé doit être intégrée à la catégorie des "incisives sciées", dont les modalités techniques du sectionnement ont été décrites en détail, sur d'autres gisements attribués au Paléolithique supérieur, par F. Poplin (1983).

À l'échelle du Massif central, cette série d'objets travaillés sur os et bois de Renne est très intéressante car des catégories d'objets jamais mentionnées à l'échelle régionale ont été identifiées (matrice à aiguilles, incisive rainurée). Une autre catégorie, comme les ciseaux, semble exister dans des assemblages publiés, sans pour autant avoir été déterminée en tant que telle (Fontana et al., 2003) et reste rare à l'échelle régionale. Enfin, les décors élaborés constitués d'incisions profondes, sans être inédits, viennent étoffer un corpus peu abondant (Bourdelle et Mazières, 1983).

\section{Caractérisation du site et des occupations}

L'étude préliminaire des vestiges et les observations de terrain nous ont permis d'envisager l'hypothèse d'une loupe de glissement pour expliquer la localisation actuelle des témoins d'occupation humaine découverts dans le talus de la route. L'abondance des restes de micromammifères, systématiquement associés au mobilier archéologique et traduisant une accumulation liée aux rapaces, suggère l'éventualité d'une position primaire du site au pied d'un escarpement orientée Ouest/Sud-Ouest, actuellement visible en contre-haut de la parcelle, à une centaine de mètres de l'accumulation. Le site des Petits Guinards doit donc être considéré à présent comme une occupation sous abri et non comme un site de plein air. L'attribution chronoculturelle constituait la seconde problématique de ce sondage puisque la datation radiocarbone obtenue en $1982(17420 \pm 330 \mathrm{BP})$ apparaissait déjà en contradiction avec l'attribution au Magdalénien supérieur 

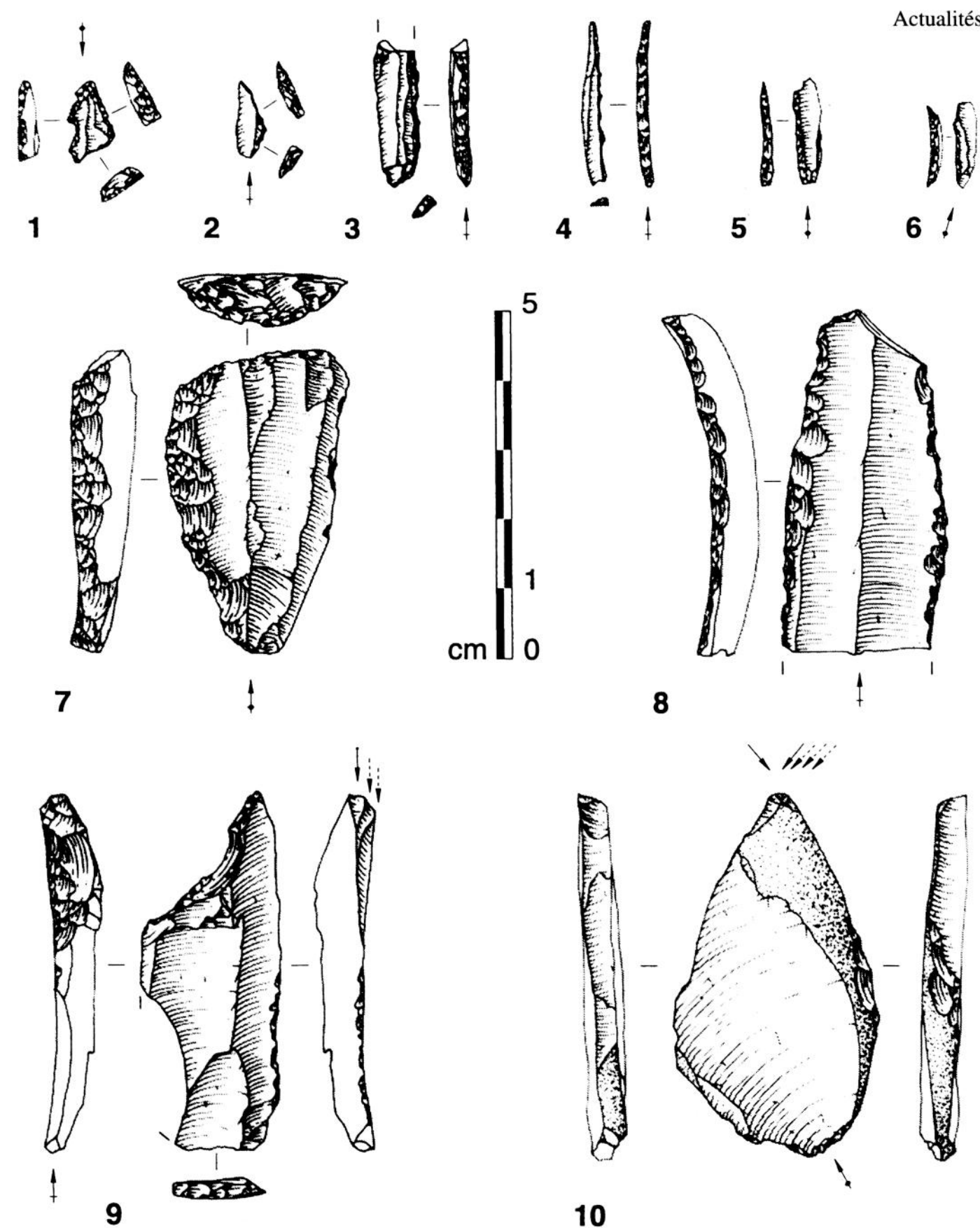

10

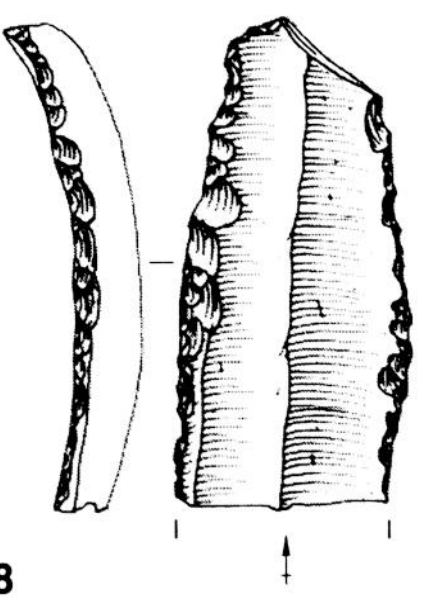

8

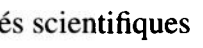




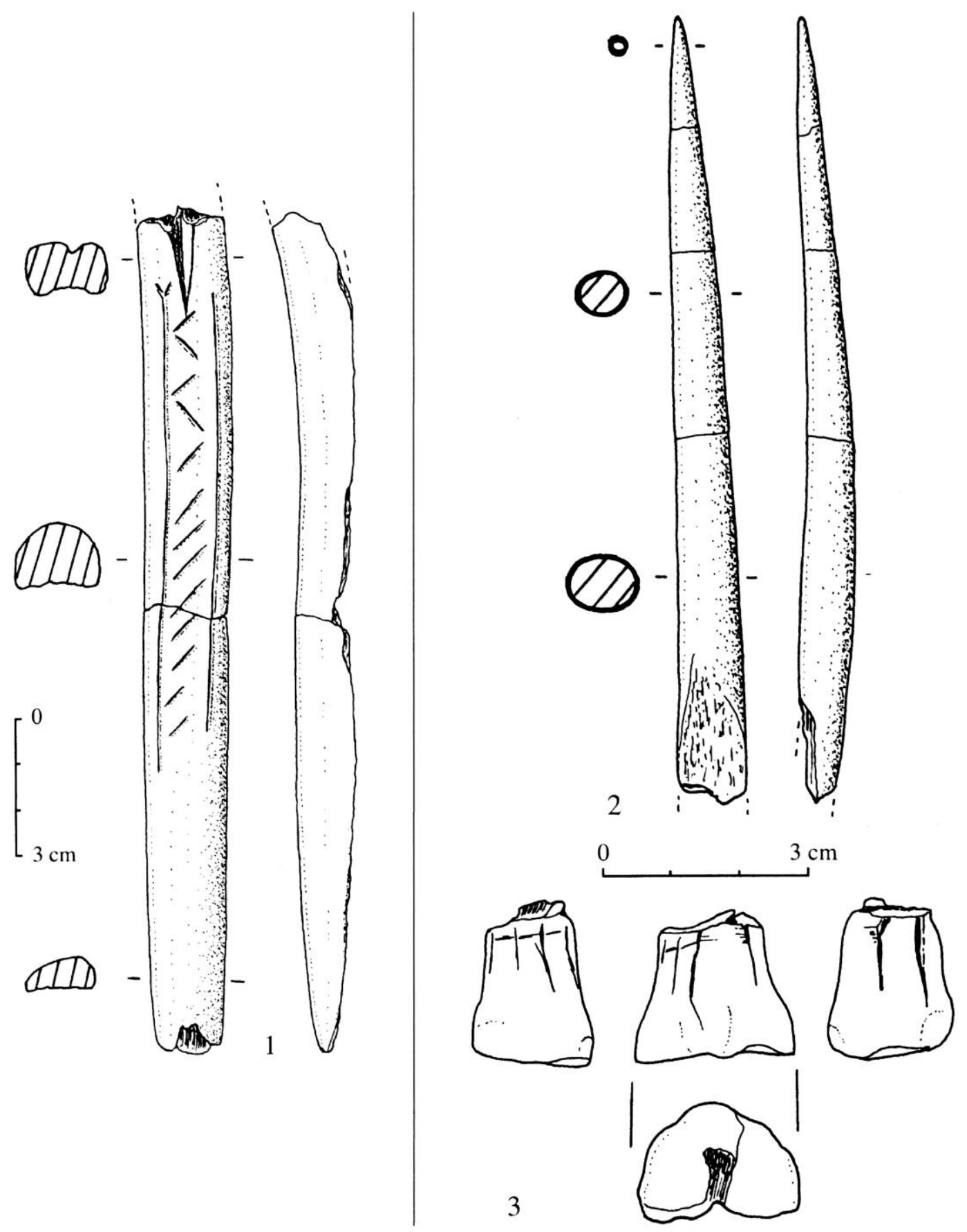

Fig. 3 - Industrie sur matières dures animales : ciseau décoré en bois de Renne (1).

Engraved Reindeer antler Chisels (I).

de l'industrie lithique. Les quatre premières datations radiocarbones (AMS) obtenues viennent de montrer que cette incohérence n'était qu'apparente puisqu'elle met en évidence au moins deux grandes périodes d'occupation du site, l'une vers $13500 \mathrm{BP}$ et l'autre vers 18000 BP. La fin du Magdalénien moyen est donc bien représentée sur ce site avec un autre faciès culturel dont l'attribution au Badegoulien ne va pas de soi dans l'état actuel des données (Fontana et al., 2003). Quoi qu'il en soit, cette première série de datations est fondamentale car elle identifie pour la première fois une occupation autour de 13500 BP dans ce secteur et elle confirme une fréquentation ancienne en livrant une date qui n'a d'équivalent que les dates récentes (inédites) des niveaux badegouliens du Blot. Enfin, si le caractère secondaire des dépôts ne fait aucun doute, le processus de mise en place (loupe de glissement) probable, associé à la cohérence stratigraphique des dates obtenues tend à confirmer une relative intégrité verticale des dépôts et du mobilier : la poursuite des études et des datations mais aussi des travaux de terrain permettront de confirmer ou non le caractère très limité des perturbations (en termes horizontal et vertical) et de les caractériser en détail. 


\section{Conclusion}

Ce nouveau sondage sur le site des Petits Guinards a livré de nombreux vestiges, variés et pour certains d'entre eux inédits à l'échelle régionale. Leur étude plus détaillée a déjà permis de mettre en évidence les particularités de ce site par rapport aux autres sites du Massif central et elle a confirmé l'importance des enjeux de l'étude de ce secteur situé au nord de la Limagne (Fontana, 1998 et sous presse). L'accent sera mis sur les deux aspects particulièrement novateurs de ce site : les activités liées à l'exploitation du bois de Renne, qui prennent une importance certaine au regard de la localisation septentrionale du site (Chauvière et Fontana, à paraître) d'une part, et la caractérisation culturelle de l'occupation la plus ancienne d'autre part. L'étude intégrée des différentes catégories de vestiges (avec un corpus augmenté par les travaux de terrain en 2003) nous permettra de comprendre le statut de ce site dans l'organisation des acquisitions à l'échelle du cycle annuel.

\section{RÉFÉRENCES BIBLIOGRAPHIQUES}

BOURDELLE Y., MAZIÈRE G. (1983) - L'art préhistorique, Les inédits de la préhistoire auvergnate, Catalogue d'exposition du Musée Bargoin, Clermont-Ferrand, 1983, p. 163-183.

Carte géologique de la France à 1/50000 - Feuille XXVI-29 (Vichy), Orléans, Bureau des Recherches Géologiques Minières, $50 \mathrm{p}$.

CHAUVIÈRE F.-X., FONTANA L. (à paraître) - L'exploitation des rennes du Blot (Haute-Loire) : entre subsistance, technique et symbolique, in V. Dujardin dir., Industrie osseuse et parure du Solutréen au Magdalénien en Europe. Table ronde sur le Paléolithique supérieur récent, Mémoire de la Société préhistorique française.

FONTANA L. (1998) - Mobilité et subsistance au Magdalénien supérieur et final en Auvergne, in J.-P. Brugal, L. Meignen, M. PatouMathis dir., Économie préhistorique : les comportements de subsistance au paléolithique, Actes du Colloque d'Antibes (oct. 1997), Antibes, APDCA, p. 373-386.

FONTANA L. (2000) - La faune du Pont-de-Longues (Les Martres-deVeyre, Puy-de-Dôme) : étude archéozoologique d'un site magdalénien de plein-air, Préhistoire du Sud-Ouest, t. 7/1, p. 41-58.

FONTANA L. (sous presse) - Territoires, mobilité et échanges au Magdalénien dans l'Aude et le Massif central (France) : approche comparative, modélisation et perspectives, Territoires, déplacements, mobilité, échanges. Actes du $126^{\circ}$ congrès du CTHS, Toulouse, 2001.

FONTANA L., LANG L., CHAUVIÈRE F.-X., JEANNET M., MAGOGA L. (2003) - Le Badegoulien et le Magdalénien du nord du Massif central : des données inattendues sur le site des Petits Guinards à Creuzier-le-Vieux (Allier, France), Préhistoire du SudOuest, t. 10/1, p. 77-93.

MAGOGA L. (1985) - Les Guinards, Creuzier le Vieux (Allier), rapport de sondage (aut. 5.09), Document dactilographié, 2 pages, Archives du service régional de l'archéologie d'Auvergne.

MASSON A. (1981) - Pétroarchéologie des roches siliceuses, intérêt en Préhistoire, Thèse de troisième cycle, Université de Lyon I, 101 p.

MAZIÈRE G. (1982) - Informations archéologiques, Circonscription Auvergne, Gallia Préhistoire, t. 25, fasc. 2, p. 361.

MAZIÈRE G. (1984) - Informations archéologiques, Circonscription Auvergne, Gallia Préhistoire, t. 27, fasc. 2, p. 309.

MAZIÈRE G. (1986) - Informations archéologiques, Circonscription Auvergne, Gallia Préhistoire, t. 29, p. 259.

POPLIN F. (1983) - Incisives de renne sciées du Magdalénien d'Europe occidentale, La faune et l'homme préhistorique. Dix ans d'étude en hommage à Jean Bouchud, Mémoire de la Société préhistorique française, t. XVI, p. 55-67.

SURMELY F., PASTY J.-F., ALIX P., DUFRESNE N., LIABEUF R., MURAT R. (2002) - Le gisement magdalénien du Pont-de-Longues (Les-Martres-de-Veyre, Puy-de-Dôme), Bulletin de la Société préhistorique française, t. LXXXXIX, p. 13-38.

Laure FONTANA UMR 6636 du CNRS

Maison méditerranéenne des Sciences de l'Homme 5, rue du château de l'horloge, BP 647 13094 Aix-en-Provence cedex 02

Laurent LANG

INRAP, 33, grande-rue, 45500 Autry-le-Chatel

François-Xavier CHAUVIÈRE

Institut de Préhistoire de l'Université de Neuchâtel

Laténium, Musée d'archéologie de Neuchâtel

Espace Paul Vouga, CH-2068 Hauterive, Suisse

Marcel JEANNET

UMR 6636 et ARPA

Université Claude Bernard - Lyon I

UFR Sciences de la Terre, 69622 Villeurbanne cedex

Lionel MAGOGA

Résidence de la Tour des Aigles

2, rue R.-Dubois

Boulevard M.-Franchet, 03200 Vichy 
